\title{
Anthrovision
}

Vaneasa Online Journal

$2.1 \mid 2014$

Anthropologie et Numérique

\section{Anthropologie et numérique: renouvellement méthodologique ou reconfiguration disciplinaire?}

Antonio A. Casilli

\section{(2) OpenEdition}

\section{Journals}

Édition électronique

URL : http://journals.openedition.org/anthrovision/626

DOI : 10.4000/anthrovision.626

ISSN : 2198-6754

Éditeur

VANEASA - Visual Anthropology Network of European Association of Social Anthropologists

Référence électronique

Antonio A. Casilli, «Anthropologie et numérique: renouvellement méthodologique ou reconfiguration disciplinaire? », Anthrovision [En ligne], 2.1 | 2014, mis en ligne le 15 septembre 2014, consulté le 19 avril 2019. URL : http://journals.openedition.org/anthrovision/626 ; DOI : 10.4000/anthrovision.626

Ce document a été généré automatiquement le 19 avril 2019

(C) Anthrovision 


\title{
Anthropologie et numérique: renouvellement méthodologique ou reconfiguration disciplinaire?
}

\author{
Antonio A. Casilli
}

1 Le numérique pose à l'anthropologie culturelle - et plus généralement aux sciences sociales - des enjeux d'ordre méthodologique et épistémologique. Il représente avant tout une manière d'ouvrir la discipline à de nouveaux sujets et domaines d'investigation. Les chercheurs contemporains portent leur regard d'anthropologues sur des pratiques émergentes (des jeux vidéos à l'usage de mobiles et tablettes, en passant par les rencontres en ligne), voire saisissent des pratiques existantes avec de nouveaux outils (entretiens non-présentiels, observations participantes en ligne, analyse de bases de données qualitatives natives du Web, etc.).

2 Par delà l'émergence d'une anthropologie du numérique (c'est-à-dire des pratiques et des représentations qui entourent l'usage des TIC) et d'une anthropologie par le numérique (dans laquelle les technologies instrumentent les recherches dans le domaine) on peut légitimement se poser la question de savoir si nous assistons à la naissance d'une anthropologie numérique, à envisager comme une discipline à part entière. D'autres domaines de recherche, telles les sciences humaines, ont récemment vécu un sursaut épistémologique et une consolidation méthodologique avec l'essor des digital humanities. Des évolutions comparables se dessinent-elles pour l'anthropologie?

3 L'un des premiers auteurs à avoir analysé les significations des usages des médias sociaux en anthropologie, Michael Wesch, les résume en disant que face à la création d'un nouveau panorama médiatique, de nouvelles temporalités de la production et de la distribution scientifique se mettent en place. Ceci entraîne, selon l'auteur, des modalités de collaboration et de mise en commun du travail de recherche, qui finalement imposent des formes de participation à la communauté scientifique distinctives ${ }^{1}$. L'internet rend-il plus simple la constitution de bases de connaissances en anthropologie, sans se soucier des barrières entre experts et profanes, entre observateurs et sujets observés? Dans ce 
contexte, comment se positionne le chercheur par rapport à son objet d'étude? Dans une certaine mesure le numérique aide à devancer toute prétention que l'anthropologie a pu à un certain moment avoir à fournir une médiation objective des faits culturels via un savoir expert. La balance entre les méthodologies emic (centrées sur des significations culturelles spécifiques aux groupements humains étudiés) et etic (centrées sur des représentations indépendantes de celles portées par les acteurs), s'inverse en la faveur de la première ${ }^{2}$. $\mathrm{Si}$, via le Web, les résultats des analyses se rendent accessibles, commentables, partageables par les sujets enquêtés, ils ne cessent jamais d'être d'une certaine façon « autochtones " parce que culturellement ancrés dans la réalité de terrain.

Mais l'exaltation des vertus démocratiques des savoirs en ligne tourne vite en rond et s'avère acritique, si l'on ne prend pas en compte les fractures et les inégalités d'accès aux contenus en ligne. Le risque ici est de faire fi des compétences nécessaires pour analyser et appréhender une réalité sociale - compétences qui deviennent encore plus sophistiquées quand les pratiques d'enquête s'articulent avec des dispositifs sociotechniques. Les savoirs experts des chercheurs ne sont donc pas automatiquement remplacés par des savoirs participatifs du numérique.

5 En s'éloignant de la rhétorique facile de l'internet libérateur des savoirs, on peut se limiter à une définition minimaliste de l'anthropologie numérique, qui se concentrerait sur ses modalités spécifiques de médiation et de restitution des corpus d'entretiens, des notes de terrain et des résultats des analyses. La diffusion de ces matériaux à l'aide de plateformes de partage social audio et vidéo telles Soundcloud ou YouTube constitue une manière de valoriser la forme traditionnelle de l'archive. Ce sont surtout les pratiques documentaires en anthropologie qui s'en retrouvent reformées. Des liens étroits avec les études des médias, l'informatique ou avec les cultural analytics se tissent. Depuis quelques temps, les travaux du réseau thématique Media Anthropology, animé à l'EASA par John Postill, interrogent les promesses et les contraintes de ces croisements disciplinaires. Une réflexion plus enraciné dans la pratique anthropologique contribue aussi, selon Postill, à mettre en question certaines catégories héritées des sciences sociales, telles la notion de "communauté » ou la dichotomie «local/global», qui risquent d'être imprudemment considérées comme de nouveaux paradigmes des études sur le numérique ${ }^{3}$. Une attention accrue aux conditions matérielles de constitution de ces archives (analysant autant les dispositifs que les acteurs et les intérêts locaux qui entrent en jeu) ${ }^{4}$ permet aussi de contrebalancer les enthousiasmes parfois simplistes qui animent le monde de la recherche sur Internet.

6 La mise en ligne des sources et des analyses en anthropologie constitue aussi une étape intermédiaire entre les observations et leur restitution sous forme de publication scientifique. Les revues scientifiques et les modalités de validation par les pairs ne disparaissent pas, mais on assiste à un glissement progressif vers un modèle de publication "open ». Les cahiers de terrain sous forme de blogs, les corpus de sources sous forme de galeries d'images, les documentaires et les archives sonores en ligne sont autant d'exemples d'un type de publication scientifique en anthropologie, répondant à des critères de qualité de la recherche mais en même temps d'accessibilité de cette dernière par des publics spécialisés et non spécialisés. Si ceci ne représente pas dans l'absolu une nouveauté (les anthropologues ont longuement expérimenté les possibilités offertes par les dispositifs d'enregistrement audio et vidéo des pratiques humaines), il constitue du moins une manière de se composer avec les règles du publish or perish, de la 
publication à tout prix, en dépassant les contraintes commerciales régissant les revues et les maisons d'édition gérées par des institutions et des groupes internationaux.

\section{NOTES}

1. Michael Wesch (2007) What is Web 2.0? What Does It Mean for Anthropology? Lessons From an Accidental Viral Video. Anthropology News, vol. 48, no. 5, p. 30-31.

2. Pour une discussion de la controverse emic vs. etic dans l'anthropologie anglophone, v. JeanPierre Olivier De Sardan (1998) Émique, L'Homme, vol. 38, no. 147, p. 151-166.

3. John Postill (2011) Localizing the Internet: An Anthropological Account, Berghahn Books.

4. John Postill (2007) The elements of media: a field theoretical exploration. Invited presentation to the German Anthropological Association (DGV) conference, Martin Lüther University, Wittenberg-Halle, 4 October 2007 < http://www.dgv-tagung2007.de/workshop-05/john-postillthe-elements-of-media-a-field-theoretical-exploration_/index.htm > [dernier accès 04 Jan. 12]

\section{RÉSUMÉS}

Cette contribution pose les questions soulevées par l'utilisation des technologies numériques au sein des sciences humaines et sociales. Le numérique va-t-il transformer les pratiques et les méthodes au sein de ces disciplines ou favoriser la création de nouvelles disciplines et de nouvelles méthodes?

This contribution raises questions around the utilisation of the digital technologies in Social Sciences and Humanities. Could Digital technologies transform methodologies and practices in these disciplines or could it foster the creation of new disciplines and within new methodologies?

Esta contribución plantea problemáticas acerca del uso de las tecnologías digitales en la Ciencias Sociales y las Humanidades. ¿Las tecnologías digitales van transformar las metodologías y prácticas de éstas disciplinas o van a promover la creación de nuevas disciplinas y nuevas metodologías?

\section{INDEX}

Mots-clés : technologies numériques, méthodologies, innovation

Keywords : digital technologies, methodology

Palabras claves : tecnologías digitales, metodología, innovación 
AUTEUR

ANTONIO A. CASILLI

LTCI Télécom ParisTech/CNRS UMR5141 